\title{
Optic pathway glioma
}

INSERM

\section{Source}

INSERM. (1999). Orphanet: an online rare disease and orphan drug data base. Optic pathway glioma. ORPHA:2086

Optic pathway glioma (OPG) is a benign tumor that develop along the optic nerve (chiasm, tracts, and radiations) characterized by impairment or loss of vision and may be accompanied by diencephalic symptoms such as reduced growth and alteration in sleeping patterns. OPG are often linked to neurofibromatosis type 1 (NF1, see this term). 